\title{
Experiments on Seismic Metamaterials: Molding Surface Waves
}

\author{
S. Brûlé, ${ }^{1}$ E. H. Javelaud, ${ }^{1}$ S. Enoch, ${ }^{2}$ and S. Guenneau ${ }^{2}$ \\ ${ }^{1}$ Ménard, 91620 Nozay, France \\ ${ }^{2}$ Aix-Marseille Université, CNRS, Centrale Marseille, Institut Fresnel, UMR 7249, 13013 Marseille, France
}

(Received 18 May 2013; published 31 March 2014)

\begin{abstract}
Materials engineered at the micro- and nanometer scales have had a tremendous and lasting impact in photonics and phononics. At much larger scales, natural soils civil engineered at decimeter to meter scales may interact with seismic waves when the global properties of the medium are modified, or alternatively thanks to a seismic metamaterial constituted of a mesh of vertical empty inclusions bored in the initial soil. Here, we show the experimental results of a seismic test carried out using seismic waves generated by a monochromatic vibrocompaction probe. Measurements of the particles' velocities show a modification of the seismic energy distribution in the presence of the metamaterial in agreement with numerical simulations using an approximate plate model. For complex natural materials such as soils, this large-scale experiment was needed to show the practical feasibility of seismic metamaterials and to stress their importance for applications in civil engineering. We anticipate this experiment to be a starting point for smart devices for anthropic and natural vibrations.
\end{abstract}

DOI: 10.1103/PhysRevLett.112.133901

PACS numbers: 41.20.Jb, 42.25.Bs, 42.70.Qs, 43.20.Bi

In 1987, the groups of E. Yablonovitch and S. John reported the discovery of stop band structures for light $[1,2]$. Photonic crystals (PCs) have since then held their promises with numerous applications ranging from nearly perfect mirrors for incident waves-whose frequencies fall within the stop bands of the PCs-to high- $q$ cavities for PCs with structural defects [3]. The occurrence of stop bands in PCs also leads to anomalous dispersion whereby dispersion curves have a negative or vanishing group velocity. Dynamic artificial anisotropy, also known as all-angle-negative-refraction [4-7], allows for focusing effects through a PC, as envisioned 45 years ago by Veselago [8]. With the advent of electromagnetic metamaterials $[9,10]$, Pendry pointed out at the turn of the 21 st century that the image through the Veselago lens can be deeply subwavelength [11], and exciting effects were demonstrated, such as simultaneously negative phase and group velocities of light [12], invisibility cloaks [13], and a tailored radiation phase pattern in epsilon-near-zero metamaterials $[14,15]$. In parallel, research papers in phononic crystals provided numerical and experimental evidence of the filtering [16] and focusing properties [17] of acoustic waves, including Rayleigh wave attenuation in marble quarry with air holes displaying $\mathrm{kHz}$ stop bands [18] and similar filtering effects in a microstructured piezoelectric for $\mathrm{MHz}$ surface waves [19]. Localized resonant structures for elastic waves propagating within threedimensional cubic arrays of thin coated spheres [20] and

Published by the American Physical Society under the terms of the Creative Commons Attribution 3.0 License. Further distribution of this work must maintain attribution to the author(s) and the published article's title, journal citation, and DOI. fluid-filled Helmholtz resonators [21] paved the way for acoustic analogues of electromagnetic metamaterials [20-23], including elastic cloaks [24-26]. The control of elastic wave trajectories in thin plates was reported numerically $[27,28]$ and experimentally $[29,30]$ during the last few years, which prompted civil engineers at the Ménard company [31] to explore potential routes toward metamaterials for surface seismic waves [32,33] in civil engineering applications [34].

In civil engineering works, Rayleigh waves are generated by anthropic sources, such as explosions, tool impacts, or vibrations (e.g., sledgehammer, pile driving operations, vibrating machine footing, or dynamic compaction, the latter being a ground-improvement technique that densifies soils and fills by using a drop weight). In earthquake engineering, the amplification of seismic waves, namely site effects, may strengthen the impact of an earthquake in specific areas (e.g., Mexico in 1985). When seismic waves propagate through soft superficial alluvial layers or scatter on strong topographic irregularities, refraction or scattering phenomena may indeed strongly increase the amplitude of the ground motion. At the scale of an alluvial basin, seismic effects involve various phenomena, such as wave trapping, resonance of the whole basin, propagation in heterogeneous media, and the generation of surface waves at the basin edges $[32,33]$. Due to the surface wave velocity in superficial and underconsolidated recent material (less than $100 \mathrm{~m} / \mathrm{s}$ to $300 \mathrm{~m} / \mathrm{s}$ ), wavelengths of surface waves induced by natural seismic sources or construction work activities are shorter than those of earthquake generated direct $P$ (primary, i.e., longitudinal compressional) and $S$ (secondary, i.e., transverse shear) waves (considering the 0.1 to $50 \mathrm{~Hz}$ frequency range), from a few meters to a few hundreds of meters. These 
are of similar length to that of buildings, therefore leading to potential building resonance phenomena in the case of earthquakes. In 1968, Woods realized in situ tests with a 200 to $350 \mathrm{~Hz}$ source to show the effectiveness of isolating circular or linear empty trenches [35]. With the same geometry, these results were compared in 1988 with numerical modeling studies performed by Banerjee [36]. Here, we study the possibility to realize seismic metamaterials not only for high-frequency anthropic sources but for the earthquake frequency range, i.e., 0.1 to $50 \mathrm{~Hz}$. A full-scale experiment was conducted during the summer of 2012. The preliminary objective of this seismic field test was to point out analogies with control of electromagnetic $[10,14,15]$ and acoustic $[16,17]$ waves by a quantitative approach.

In theory, it seems realistic to influence seismic waves passing through an artificial anisotropic medium. However, soils possess particular characteristics, such as nonelastic behavior, a high rate of signal attenuation, large-scale heterogeneity, etc. These various uncertainties and our objective of realistic input values for the simulations required in situ preliminary tests to adjust the soil parameters, such as shear modulus, quality factor, etc. A test zone with thick homogeneous silty clay soil was selected near the alpine city of Grenoble (France) in August 2012 (Fig. 1). The depth of the basin with similar deposits is up to $200 \mathrm{~m}$. The Rayleigh wave velocity was first estimated to be $78 \mathrm{~m} / \mathrm{s}$ by the preliminary seismic test by pointing the wave time arrival at various offsets from the source. Numerical simulations with finite elements were performed to predict the stop-band frequency for elastic surface waves. The seismic metamaterial test is however really challenging to model in full (threedimensional Navier equations in unbounded heterogeneous media). We therefore opted for an asymptotic model, which only captures the wave physics at the air-soil interface, and we do not claim to have a complete understanding of the elastic wave propagation. We consider the following approximate Mindlin plate model [37] for time-harmonic surface flexural waves, where the displacement field is sought in the form $z[u(x, y), v(x, y), 0]^{T}+[0,0, \Psi(x, y)]^{T}$, with $T$ the transpose and $u(x, y)=\mathfrak{R}[u(x, y, t) \exp (-1 \omega t)]$, (a)

(b)

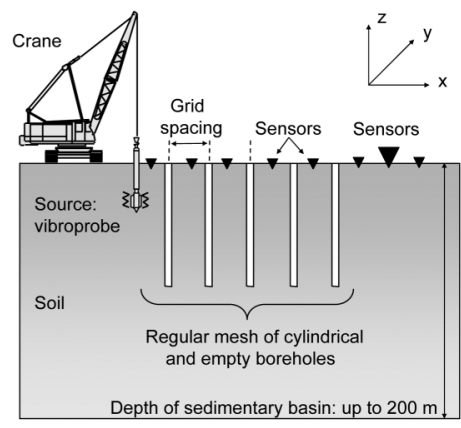

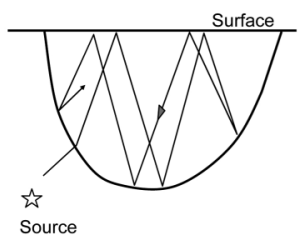

FIG. 1. Schematics of (a) a seismic wave in an alluvium basin and (b) the seismic testing device cross section in the $x$ - $z$ plane (see Fig. 3 for a photograph of the experiment). $v(x, y)=\Re[v(x, y, t) \exp (-1 \omega t)]$ and $\Psi(x, y)=\Re[\Psi(x, y, t) \times$ $\exp (-1 \omega t)]$, where $\omega$ is the angular flexural wave frequency, $x, y, z$ are the space variables, and $t$ is the time variable,

$$
\begin{gathered}
\kappa h\left(\nabla \cdot \mu \nabla \Psi+\mu \frac{\partial u}{\partial x}+\mu \frac{\partial v}{\partial y}\right)+\rho h \omega^{2} \Psi=0, \\
\nabla \cdot D(1-\nu) \nabla u+\frac{\partial}{\partial x}\left[D(1+\nu)\left(\frac{\partial u}{\partial x}+\frac{\partial v}{\partial y}\right)\right] \\
-2 \kappa \mu h\left(\frac{\partial \Psi}{\partial x}+u\right)+\frac{\rho h^{3}}{6} \omega^{2} u=0, \\
\nabla \cdot D(1-\nu) \nabla v+\frac{\partial}{\partial y}\left[D(1+\nu)\left(\frac{\partial u}{\partial x}+\frac{\partial v}{\partial y}\right)\right] \\
-2 \kappa \mu h\left(\frac{\partial \Psi}{\partial y}+v\right)+\frac{\rho h^{3}}{6} \omega^{2} v=0 .
\end{gathered}
$$

In these coupled equations, $\kappa, \mu, \rho$, and $\nu$ are Mindlin's normalization constant $\left(\pi^{2} / 12\right.$ [37]), the shear modulus, the mass density, and the Poisson ratio, respectively. Note that $h$ is the plate thickness, $D$ is the plate rigidity, and $\mu=E /[2(1+\nu)]$, where $E$ is Young's modulus.

A single fourth-order partial differential equation can be derived for $\Psi$ [the amplitude of displacement along the $z$ axis in Fig. 1(b)] by eliminating $u$ and $w$. When rotary inertia terms and transverse shear-deformation terms are omitted (pure bending), this leads to the Kirchhoff-Love approximation for thin plates which retains its form under geometric transforms [27],

$$
\rho^{-1} \nabla \cdot\left[E^{1 / 2} \nabla \rho^{-1} \nabla \cdot\left(E^{1 / 2} \nabla \Psi\right)\right]-\omega^{2} \frac{\rho h}{D} \Psi=0 .
$$

In the soil $\rho=1500 \mathrm{~kg} / \mathrm{m}^{3}$, and in bored holes $\rho=1.2 \mathrm{~kg} / \mathrm{m}^{3}$. The diameter of the inclusions is $0.32 \mathrm{~m}, h$ is the plate thickness (assumed to be $5 \mathrm{~m}$ ), and $D$ is the plate rigidity, which in the present case is $D=E h^{3} /\left(12\left(1-\nu^{2}\right)\right)$. From the analysis of the investigations carried out on site, the soil characteristics implemented in the model are $E=100 \mathrm{MPa}$ and $\nu=0.3$. In the finite-element model solved with the COMSOL MULTIPHYSICS software [38], we set Floquet-Bloch boundary conditions on either sides of a periodic cell (in the $x-y$ plane) of side length $d=1.73 \mathrm{~m}$ in order to compute the dispersion diagram shown in Fig. 2 using Eq. (2). We note in this approximate thin-plate model that the dispersion of Rayleigh surface waves is overestimated, but some additional computations using the more accurate Mindlin plate model (1) (which can be seen in Fig. 5 of the Supplemental Material [40]) confirm the main features predicted in Fig. 2, such as frequency ranges for partial stop bands. We further set perfectly matched layers [making use of the nice behavior of Eq. (2) under geometric transforms [27]] on either boundaries of the domain in order to account for the unbounded domain in the $x-y$ plane for the simulation shown in the inset of Fig. 2. The simulation shows that a 


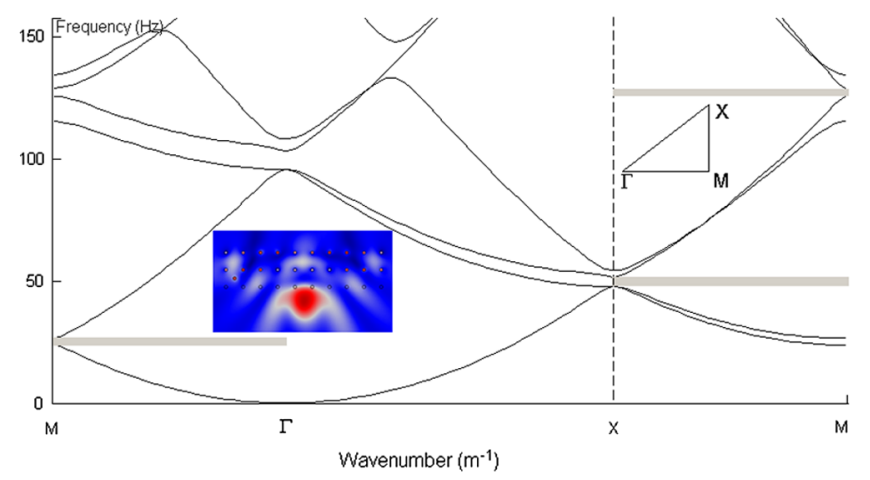

FIG. 2 (color online). Simulated dispersion curves [frequency versus Bloch wave number describing the reduced Brillouin zone of vertices $\Gamma=(0,0), X=(\pi / d, \pi / d), M=(\pi / d, 0)]$ for a periodic plate of pitch $d=1.73 \mathrm{~m}$ and thickness $5 \mathrm{~m}$, with inclusions of diameter $0.32 \mathrm{~m}$ and density about $1 / 1000$ that of the surrounding medium (soil). The inset shows the plot of flexural wave [i.e., displacement in the $x-z$ plane in Fig. 1(b)] intensity for a forcing at $50 \mathrm{~Hz}$ (frequency in the second partial stop band along $X M$ ), which is located as in Fig. 4.

seismic source frequency at $50 \mathrm{~Hz}$ is located inside the first partial stop band in Fig. 2, which confirms the stop band origin of the wave reflection. This opens a whole range of potential applications, such as seismic wave shields or wave guides [39], in a way similar to what phononic stop band materials achieve for sound [16].

A test zone consisting of a regular mesh of vertical cylindrical voids was carried out on the selected site. We set up the experiment (Fig. 3) according to the results of the simulation. The frequency of the vibrating source in the

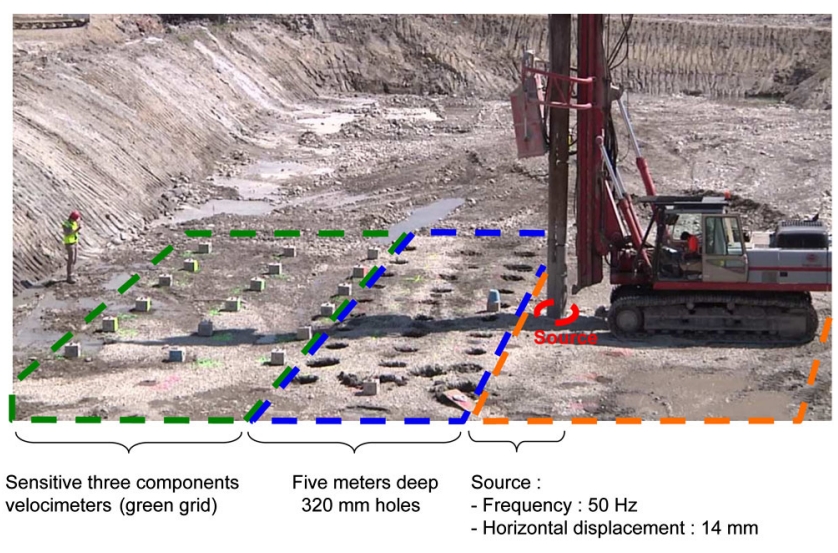

FIG. 3 (color online). Photograph of the seismic metamaterial experiment from Ménard company (see the Supplemental Material [40] for more photos and a movie [41]). The three dashed perimeters account for the location of sensors [measuring the three components of wave velocity (green area on this photograph)], seismic metamaterial [5 $\mathrm{m}$ deep self-stable holes of diameter $0.32 \mathrm{~m}$ with center-to-center spacing of $1.73 \mathrm{~m}$ (blue area)], and rotating source (a vibrating probe set on a crane) with a horizontal displacement of $0.014 \mathrm{~m}$ generating an elastic wave at frequency $50 \mathrm{~Hz}$. experiments is $50 \mathrm{~Hz}$, with $0.014 \mathrm{~m}$ of lateral amplitude in the $x-y$ plane, which should therefore lead to a very strong reflection of surface elastic waves by the large-scale metamaterial (see inset in Fig. 2). The experimental mesh is made of three discontinuous lines of ten boreholes $0.32 \mathrm{~m}$ in diameter. The length of columns is about $5 \mathrm{~m}$ and the mesh spacing is $1.73 \mathrm{~m}$. To capture the energy field, we set 20 three-component velocimeters $\left(v_{x}, v_{y}, v_{z}\right)$ with a corner frequency of $4.5 \mathrm{~Hz}(-3 \mathrm{~dB}$ at $4.5 \mathrm{~Hz})$ electronically corrected to $1 \mathrm{~Hz}$. The sensors were used simultaneously with a common time base. In order to map completely the energy field, the sensors were set four times on site before and after carrying out the boreholes.

They are the two sensors in Fig. 4(a), which have green frames (see also Fig. 3 of the Supplemental Material [40]): $A 1$ is the sensor right above the source; $B 1$ is just on the left side of the boreholes. These sensors $A 1$ and $B 1$ were kept at the same position during the whole experiment. Due to the strong soil attenuation, the probe is close to the boreholes' mesh $(1.5 \mathrm{~m})$. Each record is about two minutes long and can be divided into three parts: a preliminary experimental part, a record of ambient vibration noise, followed by the

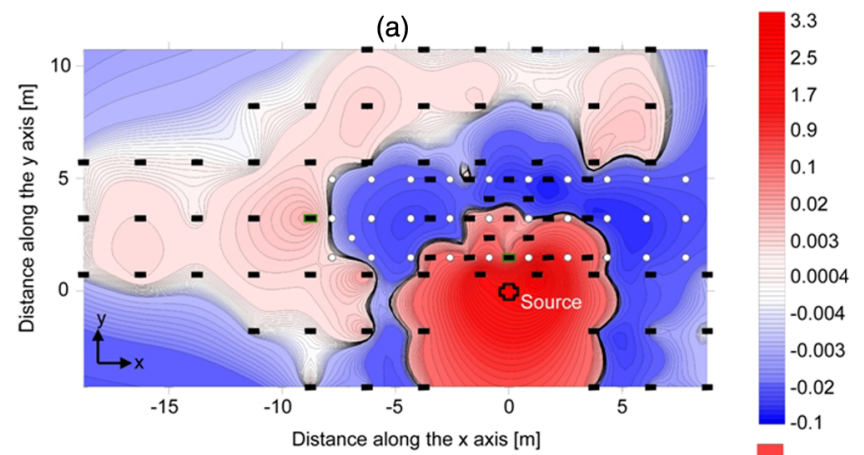

(b)

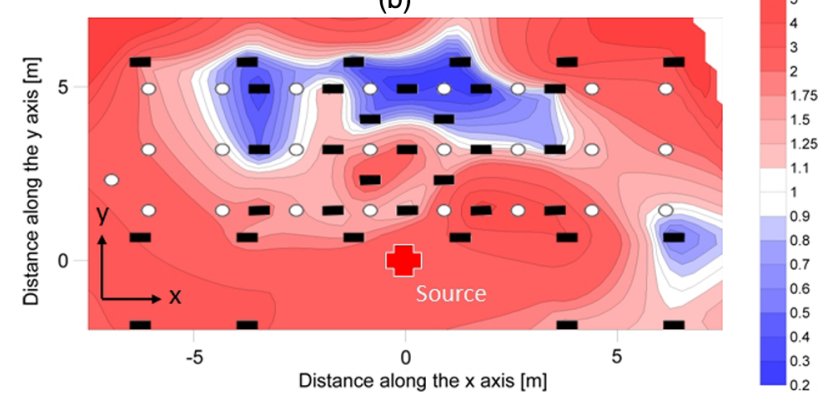

FIG.4 (color online). Measurements for a monochromatic source. Experimental results' map after interpolation between sensors: (a) the difference $\left(J_{2}-J_{1}\right)$ and (b) ratio $\left(J_{2} / J_{1}\right.$, with image magnification on the metamaterial) of the measured energy field (arbitrary units) after $\left(J_{2}\right)$ and before $\left(J_{1}\right)$ carrying out the boreholes. Note that the dark blue region in panel (b) has 5 times less elastic energy after we carried out the boreholes. Note also the small values of $J_{1}$ about 10 meters away from the source (viscoelastic soil) make $J_{2} / J_{1}$ artificially high on the upper edge of the map, and should be disregarded. Black rectangles symbolize sensors, white circles symbolize the holes, and the red cross symbolizes the source. 
probe starting phase during which the frequency of the generated signal increases up to $50 \mathrm{~Hz}$ and the probe is set in place; the experimental phase itself, about one minute long; and a post-experiment part, during which the mechanical probe stops and dies out with ambient vibration noise. For each grid, the effective experimental phase length is determined for the fixed sensor $A 1$. For each sensor grid, we have sampled a steady-state vibration duration recorded by the sensor $A 1$. It is defined as the aforementioned experimental length reduced by ten seconds at both ends to ensure that any effect on the ignition and switching off of the probe are well removed. The signals' average energy per second is then computed for each sensor. Twenty sensors were used simultaneously with a common time base. In order to map completely the energy field, the sensors were set four times on site [green, blue, pink, and orange grids in Fig. 3(a) of the Supplemental Material [40]] before and after carrying out the boreholes (Fig. 3 in [40]). These are consecutively normalized by the $A 1$ 's energy per second, so as to reconstruct a uniform energy field over the whole experimental area. The boreholes' effect on the energy field is given Fig. 4, where panel (a) shows the difference $J_{2}-J_{1}$ of the measured energy field after $\left(J_{2}\right)$ and before $\left(J_{1}\right)$ carrying out the boreholes, whereas panel (b) shows the ratio $J_{2} / J_{1}$ with image magnification on the seismic metamaterial. These two maps are displayed with interpolation between sensors, and one should note that small values of elastic energy far from the source (i.e., $10 \mathrm{~m}$ away) create the illusion of high elastic energy on the upper edge of panel (b).

According to the $50 \mathrm{~Hz}$ source, the wavelength of the signal propagated at the source is $1.56 \mathrm{~m}$, and it is comparable to the $1.73 \mathrm{~m}$ separating each borehole's center, which means we should be not too far off the Bragg regime. The center of the vibroprobe is located $1.5 \mathrm{~m}$ away from the first line of the grid, along the direction perpendicular to the slab. In this soft soil, the attenuation may be significant. We verify that the signal-to-noise ratio is over 2 at $10 \mathrm{~m}$ from the source. Figure 4(a) shows a concentration of energy in a $5 \mathrm{~m}$ radius zone centered around the vibroprobe, strongly attenuated towards the structure, which is in good agreement with the inset in Fig. 2. In this area and near the source, the difference between the measured energy field after and before carrying out the boreholes can reach twice the initial value, confirming a strong reflection of surface waves by the seismic metamaterial. The signal hardly exceeds the second row of boreholes in Fig. 4, showing the efficiency of this device for this geometry and a $50 \mathrm{~Hz}$ source in soft soils.

In this Letter, an approximate plate model (which behaves nicely under geometric transforms unlike other elastic wave equations) was used in the design of a structured soil consisting of clay with $5 \mathrm{~m}$ deep boreholes shielding surface elastic waves around $50 \mathrm{~Hz}$. This plate model has been experimentally validated, which opens a route to transformation-based designs of other, more ambitious designs of seismic metamaterials such as cloaks detouring surface elastic waves in soils with similar characteristics. The fact that the elastic energy is 2.3 times larger at the source point when it is in presence of the metamaterial [see Fig. 4(a) and the inset in Fig. 2] is reminiscent of the local density of states obtained for a source placed near a mirror in optics [42]. This suggests the strong potential of the large-scale periodic structure for the control of spontaneous emissions of seismic waves in a way similar to what is achieved for light in high-contrast nanoscale composite structures. However, we found that a ring-type geometry displays some concentration effect (see Fig. 7 of the Supplemental Material [40]). This numerical investigation suggests that one should be extra cautious with antiearthquake designs before some definite conclusions are drawn. (Note, for instance, that our numerical modeling does not account for viscoelastic features of the soil, which will damp the elastic signal; see Fig. 8 of [40] for preliminary numerics on that aspect.) The concept of a seismic cloak to protect a building from earthquakes would at least require in-depth analysis to achieve some judicious - but not necessarily periodicarrangement of boreholes. Other theoretical research [39] has suggested using metacylinders with side holes, which would dampen the seismic surface waves and hence reduce the amount of seismic energy reflected from the metamaterial. The dampening of the seismic waves means that some of the energy carried by those waves is transformed into another type, such as sound and heat. One could also envisage structure foundations of buildings that prevent elastic waves from propagating therein [43]. We finally point out that models of pinned plates suggest the existence of zero-frequency stop bands, which could be used in the design of ultrabroadband seismic-wave reflectors [44].

S. G. is thankful for European funding through ERC Starting Grant ANAMORPHISM. S. B. and E. J. thank MENARD's Earthquake Engineering Team.

[1] E. Yablonovitch, Phys. Rev. Lett. 58, 2059 (1987).

[2] S. John, Phys. Rev. Lett. 58, 2486 (1987).

[3] K. Srinivasan and O. Painter, Opt. Express 10, 670 (2002).

[4] R. Zengerle, J. Mod. Opt. 34, 1589 (1987).

[5] M. Notomi, Phys. Rev. B 62, 10696 (2000).

[6] B. Gralak, S. Enoch, and G. Tayeb, J. Opt. Soc. Am. A 17, 1012 (2000).

[7] C. Luo, S. G. Johnson, J. D. Joannopoulos, and J. B. Pendry, Phys. Rev. B 65, 201104 (2002).

[8] V. G. Veselago, Sov. Phys. Usp. 10, 509 (1968).

[9] J. B. Pendry, A. J. Holden, D. J. Robbins, and W. J. Stewart, IEEE Trans. Microwave Theory Tech. 47, 2075 (1999).

[10] D. R. Smith, W. J. Padilla, D. C. Vier, S. C. Nemat-Nasser, and S. Schultz, Phys. Rev. Lett. 84, 4184 (2000).

[11] J. B. Pendry, Phys. Rev. Lett. 85, 3966 (2000). 
[12] G. Dolling, C. Enkrich, M. Wegener, C. M. Soukoulis, and S. Linden, Science 312, 892 (2006).

[13] D. Schurig, J. J. Mock, B. J. Justice, S. A. Cummer, J. B. Pendry, A. F. Starr, and D. R. Smith, Science 314, 977 (2006).

[14] S. Enoch, G. Tayeb, P. Sabouroux, N. Guérin, and P. A. Vincent, Phys. Rev. Lett. 89, 213902 (2002).

[15] A. Alu, M. G. Silveirinha, A. Salandrino, and N. Engheta, Phys. Rev. B 75, 155410 (2007).

[16] R. Martinez-Sala, J. Sancho, J. V. Sanchez, V. Gomez, J. Llinares, and F. Meseguer, Nature (London) 378, 241 (1995).

[17] A. Sukhovich, B. Merheb, K. Muralidharan, J. O. Vasseur, Y. Pennec, P. A. Deymier, and J. H. Page, Phys. Rev. Lett. 102, 154301 (2009).

[18] F. Meseguer, M. Holgado, D. Caballero, N. Benaches, J. Sanchez-Dehesa, C. Lopez, and J. Llinares, Phys. Rev. B 59, 12169 (1999).

[19] S. Benchabane, A. Khelif, J.-Y. Rauch, L. Robert, V. Laude, Phys. Rev. E 73, 065601(R) (2006).

[20] Z. Liu, X. Zhang, Y. Y. Zhu, Z. Yang, C. T. Chan, and P. Sheng, Science 289, 1734 (2000).

[21] N. Fang, D. J. Xi, J. Y. Xu, M. Ambrati, W. Sprituravanich, C. Sun, and X. Zhang, Nat. Mater. 5, 452 (2006).

[22] J. Christensen, F. J. Garcia de Abajo, Phys. Rev. Lett. 108, 124301 (2012).

[23] R. V. Craster and S. Guenneau, Acoustic Metamaterials: Negative Refraction, Imaging, Lensing and Cloaking (Springer, Berlin, 2013).

[24] G. W. Milton, M. Briane, and J. R. Willis, New J. Phys. 8, 248 (2006).

[25] M. Brun, S. Guenneau, and A. B. Movchan, Appl. Phys. Lett. 94, 061903 (2009).

[26] A. N. Norris and A. L. Shuvalov, Wave Motion 48, 525 (2011).

[27] M. Farhat, S. Guenneau, and S. Enoch, Phys. Rev. Lett. 103, 024301 (2009).

[28] M. Farhat, S. Guenneau, and S. Enoch, Phys. Rev. B 85, 020301R (2012).

[29] N. Stenger, M. Wilhelm, and M. Wegener, Phys. Rev. Lett. 108, 014301 (2012).
[30] M. Dubois, M. Farhat, E. Bossy, S. Enoch, S. Guenneau, and P. Sebbah, Appl. Phys. Lett. 103, 071915 (2013).

[31] http://www.menard-web.com.

[32] S. Brûlé and E. Javelaud, in Proceedings of the 9th Annual International Conference on Urban Earthquake Engineering (Tokyo Tech CUEE, Tokyo, 2012), p. 497.

[33] S. Brûlé, E. Javelaud, S. Guenneau, S. Enoch and D. Komatitsch, in Proceedings of the 9th International Conference of the Association for Electrical, Transport and Optical Properties of Inhomogeneous Media, Marseille, 2012 (to be published).

[34] J. F. Semblat and A. Pecker, Waves and Vibrations in Soils: Earthquakes, Traffic, Shocks, Construction Works (IUSS Press, Pavia, 2009).

[35] R. D. Woods, Screening of Surface Waves in Soils, Technical Report No. IP-804, University of Michigan, 1968.

[36] P. K. Banerjee, S. Ahmad, and K. Chen, Earthquake Eng. Struc. Dyn. 16, 1041 (1988).

[37] R. D. Mindlin, J. Appl. Mech. 18, 31 (1951).

[38] http://www.comsol.com.

[39] S. H. Kim and M. P. Das, Mod. Phys. Lett. B 26, 1250105 (2012).

[40] See Supplemental Material at http://link.aps.org/ supplemental/10.1103/PhysRevLett.112.133901 for further details on the description of the experimental setup, some dispersion curves using the Mindlin plate model, some preliminary numerical results of a possible impact of soil's viscoelasticity, and a concentration effect induced by a ringtype seismic metamaterial.

[41] See Supplemental Material at http://link.aps.org/ supplemental/10.1103/PhysRevLett.112.133901 for a movie on the construction and characterization of the seismic metamaterial.

[42] L. Novotny and B. Hecht, Principles of Nano-Optics (Cambridge University Press, Cambridge, England, 2006).

[43] H. J. Xiang, Z. F. Shi, S. J. Wang, and Y. L. Mo, Smart Mater. Struct. 21, 112003, 2012)

[44] T. Antonakakis, R. V. Craster, and S. Guenneau, arXiv:1301.7653. 